\title{
Simulation Study on Horizontal Flue Speed Measurement of Boiler
}

Zhiyu Song ${ }^{1, a}$, Jiting Han ${ }^{1, b}$, Jiwei Cheng ${ }^{2, \mathrm{c}}$, Yingai $\mathrm{Jin}^{2}$, Ming $\mathrm{Li}^{2}$, Yan $\mathrm{Liu}^{2}$

${ }^{1}$ Jilin Province Electric Power Science Research Institute Co., Ltd, Changchun, 130021, China

${ }^{2}$ College of Automotive Engineering, Jilin University, Changchun, Jilin, 130022, China

ajdyszy@163.com, b41303705@qq.com

'Corresponding author:1055236456@qq.com

Keywords: Horizontal flue Point selection Numerical simulation.

\begin{abstract}
Boiler is one of the most important equipment of thermal power system. It is a very indispensable job to monitor the internal combustion of boilers. There are many ways to monitor the combustion process of the boiler, such as the negative pressure signal spectrum monitoring within the furnace, combustion noise detection, the furnace image processing technology. But the most practical and authoritative way of monitor technology is the installation of the furnace flue level point. This paper mainly introduces the numerical simulation of the determination of the location of the horizontal flue.
\end{abstract}

\section{Introduction}

The main object of this paper is - HG-1110 / 25.4-HM2 boiler in Changchun $2 \times 350 \mathrm{MW}$ Thermal Power Plant. It is the super critical boiler designed to fire Lignite Coal supplied and manufactured by the Harbin Boiler Works Co., Ltd.. For a middle reheat, supercritical pressure transformer operation, thenon-recirculating pump with the atmosphere expansion is used for the start-up system of the boiler, boiler with a single furnace, balanced ventilation, solid slag, full steel frame, full suspension structure, $\Pi$ type tight closure arrangement. A three - dimensional calculation model of the tail flue of the boiler was established by numerical simulation. The velocity field of the flue gas in the flue was studied, and the optimum velocity of the flue was found. In theory, we use the fluid flow in different sections of the mass flow is equal, so we can find the best position of speed, so as to find the best speed measurement points.

\section{The establishment of the model}

The water flue is the middle part of the boiler's economizer exit to the SCR inlet. We have simplified the horizontal flue, and established the 3D model of the horizontal flue according to the above drawings. as the picture shows:

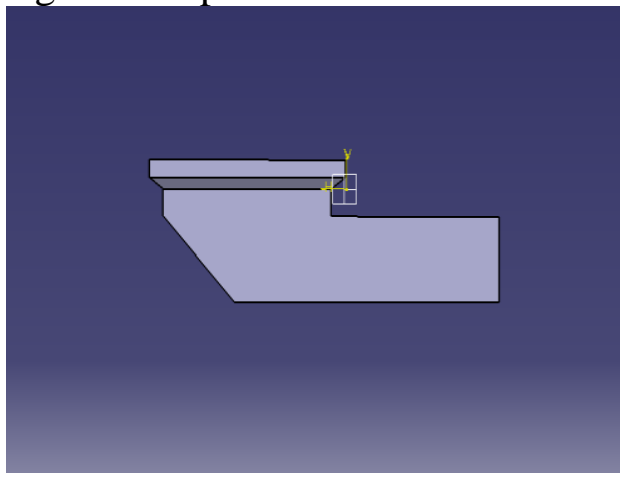

Figure1. Horizontal flue main view Figure

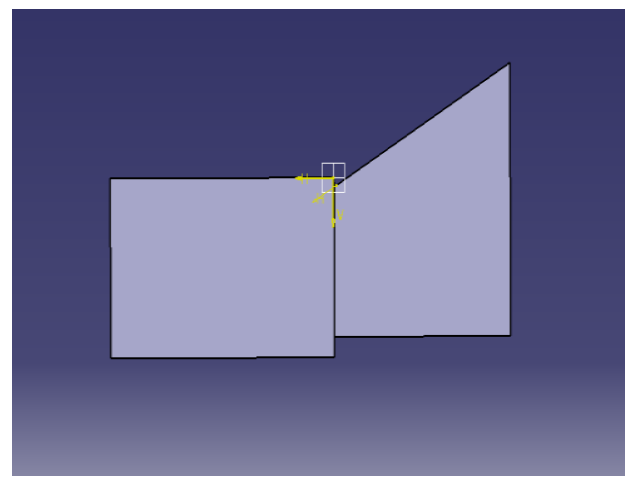

Figure2. Horizontal flue top view 


\section{Setting of boundary conditions}

Boiler in the actual operation of the process, the boiler load during the day and night, work and get off work, suburbs and cities such as the peak of power load are different. The speed of flue gas at the tail level flue is a very large range. So we set a total of six conditions for the speed boundary entrance: 5 meters per second, 7 meters per second, 9 meters per second, 11 meters per second, 13 meters per second and 15 meters per second. They are compared with actual conditions.

\section{Simulation analysis}

Horizontal flue tail is trapezoidal distribution, in the actual measurement is not good to grasp the best location of the measuring point, so we first carry out a certain numerical simulation analysis, the theoretical point of the best measurement points for the actual project to reduce a certain degree of difficulty, with a certain reference value. The following will be introduced at different speeds of the contour line cloud, the theoretical analysis of the actual speed of the process.

$5 \mathbf{m}$ per second velocity contour map analysis

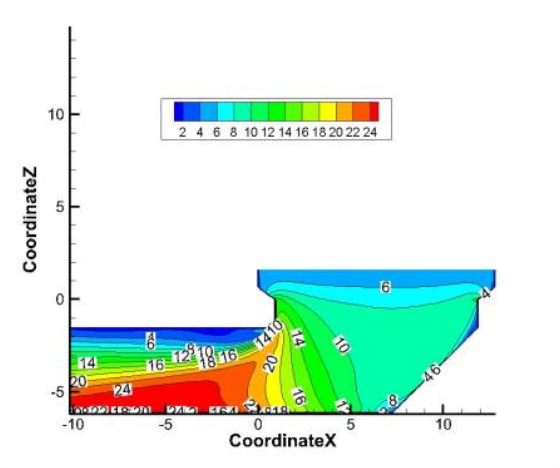

Figure 3. Velocity contour of the front view

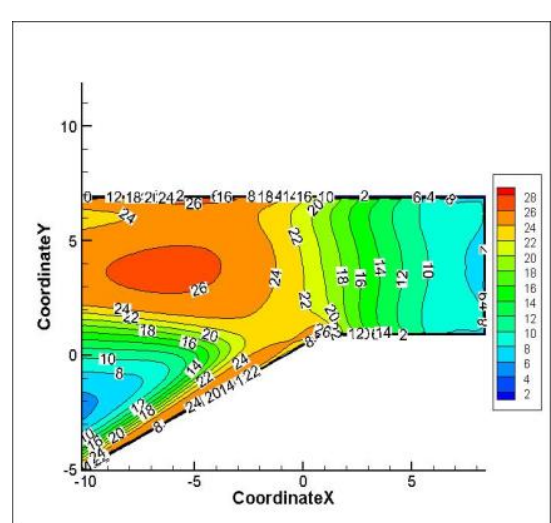

Figure4. Velocity contour map of the top view

From the above figure we can see that the velocity value is relatively small, the velocity distribution is more uniform, especially when the speed of the inlet velocity curve is more concentrated.

$9 \mathbf{m}$ per second velocity contour map analysis

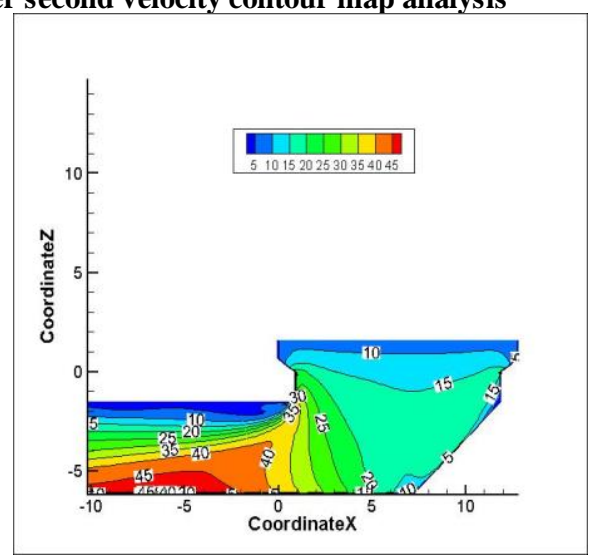

Figure 5. The velocity contour of the front view

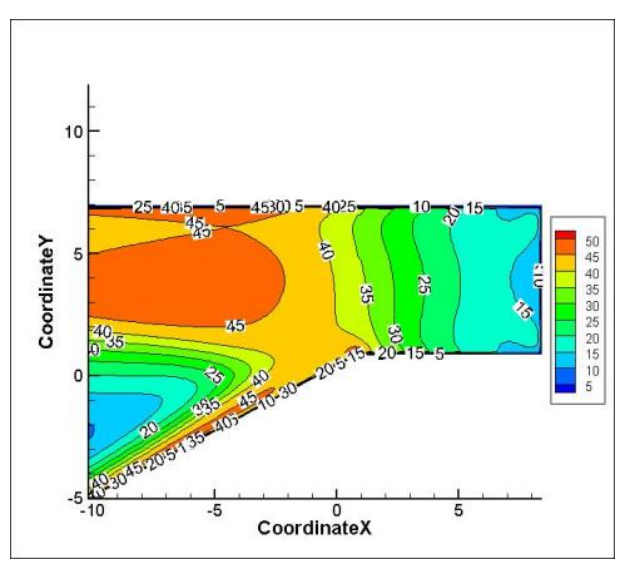

Figure 6. The velocity contour of the top view The velocity of the inlet is in the middle of the equivalent velocity, the velocity of the contour distribution is more increased, from the top view to see the corner part of the speed of the value of the larger. 
$13 \mathrm{~m}$ per second velocity contour map analysis

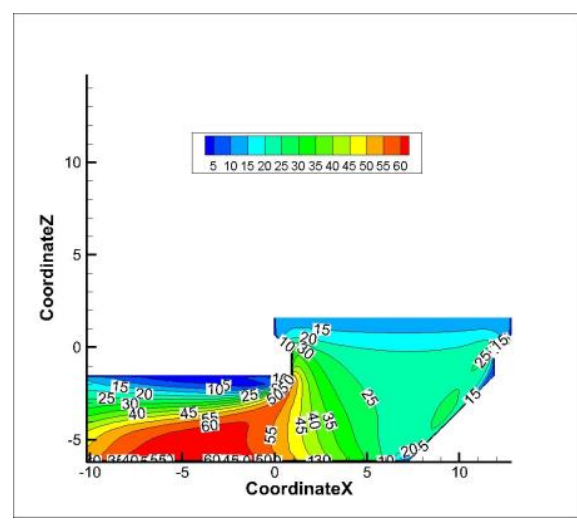

Figure 7. The velocity contour of the front view

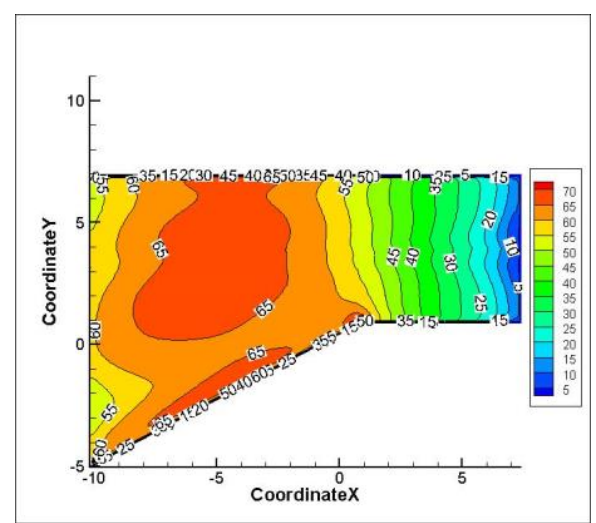

Figure 8 . The velocity contour of the top view

At the maximum speed of the entrance, the main view and the top view can be seen, the speed is significantly larger than the previous speed of the entrance, the flue speed in the tail is significantly larger than the previous, the speed of the contour line even more uniform.

The analysis of the above different velocity contours shows that:

1. From the contours of the different views of the velocity, it can be seen that the velocity of the tail flue exit is linearly increased at a certain speed as the inlet speed increases linearly with a second of two meters per meter. And from each of the front view we can see that the contours of velocity are close to the level, and the difference between them decreases gradually. Each red area of the front view is the maximum of the speed, and it can be concluded that the measuring point should be determined at these places.

2. From the contours of the top view of the different velocities, it can be seen that the velocity of the tail flue exit is linearly increasing at a certain speed as the inlet speed increases linearly with a second of two meters per meter. It is important to note that the selection of the measuring point after the increase of the speed is also possible, and it can be seen from the front view that the red area is the ideal position for selecting the best measuring point.

\section{Conclusion}

By comparing the contour map of the front view and the contour map of the top view, when the velocity is set to the linear velocity during the simulation, the exit velocity is linear velocity and the velocity distribution is reasonable Trapezoidal waist of the middle position, so select the best measuring point should be in the middle of the trapezoidal part which is theoretically the most suitable measuring point position.

\section{Acknowledgements}

The project [Improving the Combustion Mode and Security Running Control Strategy to Decrease the Pollution in Northeast of China(UK-CIAPP 201) ] was supported by the Royal Academy of Engineering under the UK-China Industry Academia Partnership Programme scheme. The authors are grateful for the support of the Royal Academy of Engineering and the UK government's Newton Fund.

This work was supported by project "Development of $\mathrm{CO}$ on-line monitoring instrument for coal-fired boiler in power plant and its application in automatic control, No.: KY-GS-17-01-05"

\section{References}

[1]. Jiaxun Liu. Radiation spectroscopy of power plant boiler combustion detection diagnosis [D]. Shanghai University of Science and Technology, 2014. 
[2]. Yun Ji, Xiaobin Huang, Jingwei Zhu. Boiler combustion state detection and integrated optimization closed-loop control system [A]. China Power Engineering Society of environmental protection technology and equipment professional committee .2012 thermal power plant pollutant purification and energy saving technology seminar Proceedings [C]. China Power Engineering Society Environmental Protection Technology and Equipment Professional Committee: 2012: 10.

[3]. Benyuan Huang. Coal and gas blending boiler combustion detection and control research [D]. Huazhong University of Science and Technology, 2010.

[4]. Luo self-learning. The introduction of radiant energy signal boiler combustion detection and optimization control [D]. Huazhong University of Science and Technology, 2007.

[5]. Lingjun Meng. Using computer digital image processing technology to detect power plant boiler burner flame [J]. Chemical Automation and Instrumentation, 2001, (02): 43-45 + 66 . 